\title{
Conservación del fruto de banano bocadillo (Musa AA Simmonds) con la aplicación de permanganato de potasio $\left(\mathrm{KMnO}_{4}\right)$
}

\section{Conservation of baby banana (Musa AA Simmonds) fruits with the application of potassium permanganate $\left(\mathrm{KMnO}_{4}\right)$}
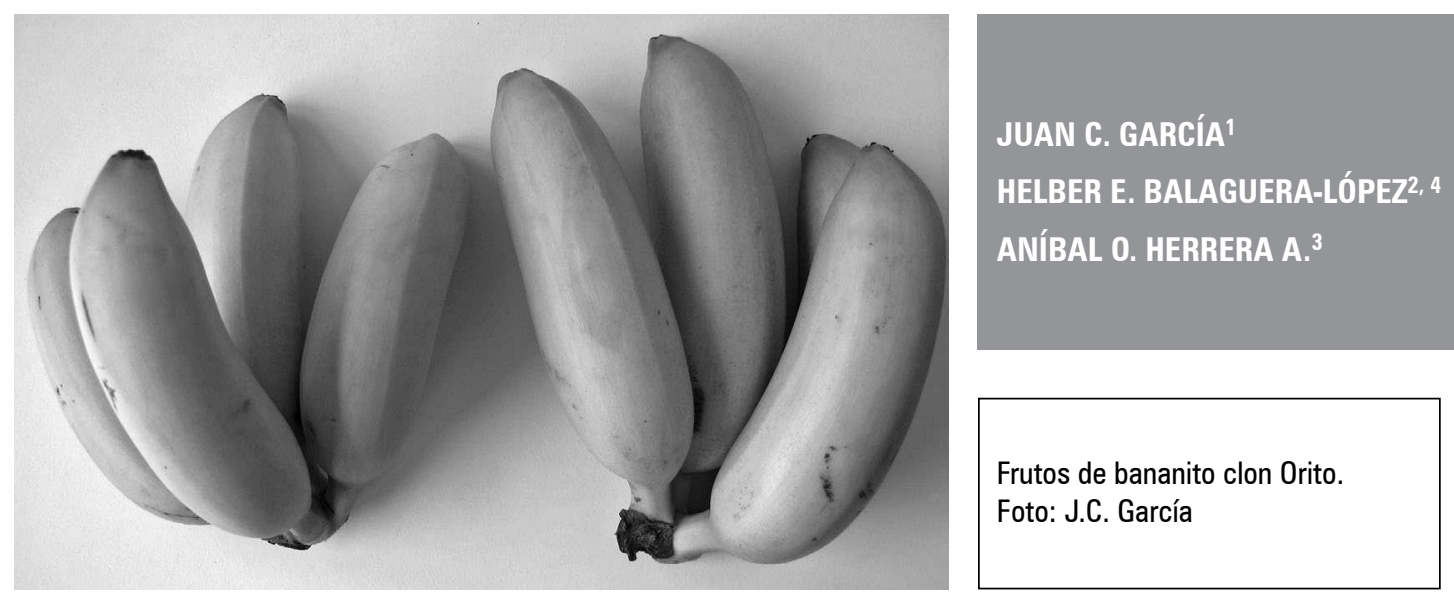

\section{RESUMEN}

El banano bocadillo es considerado uno de los productos hortofrutícolas promisorios exportables de Colombia, no obstante, es un producto altamente perecedero, por lo anterior es necesaria la evaluación de tecnologías de bajo costo que aumenten la conservación de estos frutos. El permanganato de potasio $\left(\mathrm{KMnO}_{4}\right)$ ha mostrado ser una herramienta eficaz en la conservación de diferentes frutos debido a que oxida al etileno. El objetivo de este estudio fue evaluar el efecto del $\mathrm{KMnO}_{4}$ en la conservación de frutos de banano bocadillo, para lo cual se utilizó un diseño en bloques completos al azar con arreglo factorial de $3 \times 3+1$, donde los bloques correspondieron a los tipos de arcillas (montmorillonita, caolinita, vermiculita y zeolita), el primer factor consistió en las dosis de $\mathrm{KMnO}_{4}(0,5 \% ; 1,0 \%$ y $1,5 \%$ con base en el peso fresco de los frutos) y el segundo factor fueron las dosis de arcilla $(0,5 \% ; 1,0 \%$ y $1,5 \%)$ usada como carrier del $\mathrm{KMnO}_{4}$, más un testigo absoluto, los frutos fueron almacenados durante 16 días a temperatura ambiente y periódicamente se midió el índice de color (IC), sólidos solubles totales (SST), pérdida de peso, firmeza, acidez total titulable (ATT) y relación de madurez (RM). Los resultados obtenidos muestran que la mezcla de 1\% de arcilla +1,5\% de $\mathrm{KMnO}_{4}$ favoreció la conservación de bananito, debido a que presentó menor IC (-0,64); menores SST (12,08 ${ }^{\circ}$ Brix), mayor firmeza $(70,65 \mathrm{~N})$ y menor RM $(83,67)$. Por lo tanto, existe un efecto favorable en la conservación de los frutos de bananito cuando el $\mathrm{KMnO}_{4}$ se encuentra en mayor proporción con respecto a su carrier.

Facultad de Agronomía, Universidad Nacional de Bogotá (Colombia).

2 Facultad de Agronomía, Programa de Doctorado en Ciencias Agropecuarias Área Agraria, Universidad Nacional de Colombia, Bogotá (Colombia).

3 Facultad de Agronomía, Departamento de Agronomía, Universidad Nacional de Colombia, Bogotá (Colombia).

4 Autor para correspondencia. hebalagueral@unal.edu.co 


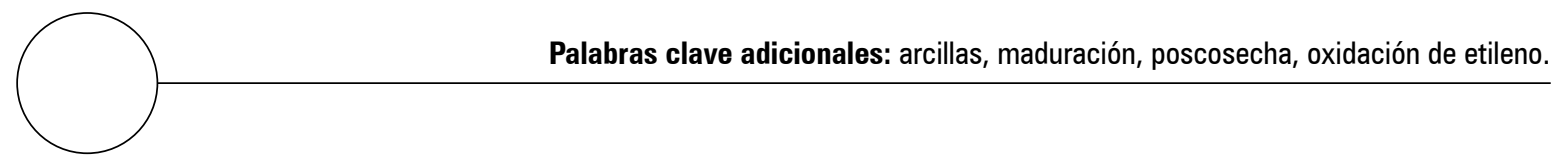

\section{ABSTRACT}

The baby banana is considered one of the promising horticultural products exported from Colombia, however, it is a highly perishable product, whereby, it is necessary to carry out an evaluation of low-cost technologies that enhance the preservation of these fruits. Potassium permanganate $\left(\mathrm{KMnO}_{4}\right)$ has proven to be an effective tool in the conservation of different fruits because this product oxidizes ethylene. The aim of this study was to evaluate the effect of $\mathrm{KMnO}_{4}$ in preserving fruits of the baby banana, for which a completely randomized design was used with a factorial arrangement of $3 \times 3+1$, wherein the blocks corresponded to the types of clays (montmorillonite, kaolinite, vermiculite and zeolite), the first factor was $\mathrm{KMnO}_{4}$ doses $(0.5 \%, 1.0 \%$ and $1.5 \%$ based on the fresh weight of fruit) and the second factor was clay doses $(0.5 \%, 1.0 \%$ and $1.5 \%)$ used as carriers of $\mathrm{KMnO}_{4}$, in addition to an absolute control. The fruits were stored for 16 days at room temperature and periodically measured for color index (CI), total soluble solids (TSS), weight loss, firmness, total titratable acidity (TTA) and maturity ratio (MR). The results showed that the mixture of $1 \%$ clay + $1.5 \% \mathrm{KMnO}_{4}$ favored baby banana conservation because this mixture had a lower CI (-0.64), lower TSS $\left(12.08^{\circ} \mathrm{Brix}\right)$, increased firmness $(70.65 \mathrm{~N})$ and had a lower MR (83.67). Therefore, there is a favorable effect on fruit conservation when $\mathrm{KMnO}_{4}$ is greater than the respective carrier.

Additional key words: clay, ripening, postharvest, ethylene oxidation.

Fecha de recepción: 11-09-2012

Aprobado para publicación: 28-11-2012

INTRODUCCIÓN

El bananito o banano bocadillo (Musa AA Simonds) es una especie frutal de la familia Musácea, es el único diploide comestible dentro del grupo Acuminata (Genoma A) (Castro, 2001). Los frutos son ricos en minerales como $\mathrm{K} y$ $\mathrm{Ca}$, carbohidratos y vitaminas $\mathrm{B}_{3}$ y $\mathrm{C}$ (Morales, 2002). El área sembrada del cultivo en Colombia reportada para el 2011 fue de 4.426 ha, con una producción de 32.108 t y un rendimiento de 7,3 t ha ${ }^{-1}$, siendo los principales departamentos productores Nariño, Putumayo, Santander y Valle del Cauca, los cuales representan el 86,0\% del área sembrada en el país (Espinal et al., 2005; Agronet, 2012). El banano bocadillo es considerado un producto hortofrutícola promisorio de exportación para Colombia. Los principales mercados de este pro- ducto son Francia (50\% de las exportaciones), Estados Unidos (34\%), Italia (6\%) y Suiza (6\%) (Legiscomex, 2008) con un valor de las exportaciones de US $\$ 2,8$ millones (Bonilla et al., 2009).

Los frutos de bananito son climatéricos y su máxima actividad respiratoria se alcanza a los $10 \mathrm{~d}$ de almacenamiento (180 $\mathrm{mg} \mathrm{CO}_{2} \mathrm{~h}^{-1} \mathrm{~kg}^{-1}$ ) a $18^{\circ} \mathrm{C}$ (Castro y Restrepo, 2004). La maduración de estos frutos es caracterizada por el incremento de la respiración y producción de etileno después de la madurez fisiológica, siendo altamente perecederos y con bajas posibilidades de almacenamiento en condiciones ambientales normales (Gutiérrez, 1997; Santosa et al., 2010). El etileno es una hormona vegetal que está relacionada 
con la maduración de los frutos de banano y bananito, debido a que regula diferentes enzimas que ocasionan ablandamiento del fruto, contribuyen a una conversión masiva de almidón a azúcares en la pulpa, incremento de la actividad polifenoloxidasa (pardeamiento enzimático), favorecen síntesis de compuestos aromáticos, degradación de clorofilas y desenmascaramiento de carotenoides (Castro et al., 2007; Kesari et al., 2007).

Debido a que la maduración de los frutos es un proceso genéticamente regulado e irreversible, la principal estrategia para la conservación de frutas y hortalizas es mantener el etileno a niveles muy bajos (Ayoub et al., 1976). En este sentido, Saltveit (1999) propone el uso de permanganato de potasio $\left(\mathrm{KMnO}_{4}\right)$ como una estrategia viable y de bajo costo para oxidar y disminuir los niveles de etileno durante el almacenamiento de frutas y hortalizas, permitiendo alargar la vida poscosecha de los mismos. Por su parte, Jiang et al. (1999) y Bhutia et al. (2011) aseguran que el uso de $\mathrm{KMnO}_{4}$ es una estrategia adecuada, principalmente en países en vías de desarrollo, debido a que no es una tecnología muy sofisticada y no implica un aumento significativo en los costos de producción.

El $\mathrm{KMnO}_{4}$ es un agente oxidante fuerte en muchas reacciones de oxidorreducción orgánicas e inorgánicas (Dash et al., 2009), cuando este compuesto entra en contacto con el etileno, lo oxida produciendo $\mathrm{CO}_{2}$ y $\mathrm{H}_{2} \mathrm{O}$, lo que ocasiona que el etileno pierda su actividad como hormona señal de los procesos de maduración de los frutos (Sammi y Masud, 2007). Al oxidar el etileno alrededor de la superficie del fruto, se genera que el gas al interior del fruto se difunda hacia el exterior, ocasionando que el etileno al interior del fruto disminuya (Brody et al., 2002), no obstante en frutos densos como manzanas, melones y tomates, este proceso se ve limitado, afectando la eficacia de dicha tecnología (Saltveit, 1999).

El $\mathrm{KMnO}_{4}$ ha mostrado eficiencia para eliminar el etileno y retrasar la maduración de diversos frutos como banano (Musa paradisiaca; Chauhan et al., 2006), mango (Mangifera indica; Illeperuma y Jayasuriya, 2002), aguacate (Persea americana; Illeperuma y Nikapitiya, 2002), níspero (Eryobotria japonica Campos et al., 2007), guanábana (Annona muricata; Chaves et al., 2007), manzana (Malus domestica; Brackmann et al., 2006), zapote (Manilkara achras; Bhutia et al., 2011), papaya (Carica papaya; Pereira et al., 2009) y banano bocadillo (Gutiérrez, 1997).

Sin embargo, para que el $\mathrm{KMnO}_{4}$ sea efectivo, debe ser adsorbido a un carrier para formar un absorbente sólido, que aumente el área de contacto, debido a que las únicas fuerzas que actúan para que ocurra el contacto absorbente-etileno son la difusión y convección natural del aire en la atmósfera. Lo anterior se ha logrado mediante la absorción a materiales minerales inertes como celita, vermiculita, alúmina, zeolita, otras arcillas, y gel sílice (Gutiérrez, 1997; Wills y Warton, 2004).

A pesar de existir estudios en la capacidad del $\mathrm{KMnO}_{4}$ de oxidar el etileno y prolongar la vida poscosecha de frutas y hortalizas, no existen estudios que establezcan las dosis adecuadas de permanganato y de su Carrier en la conservación de banano bocadillo (Wills y Warton, 2004). A partir de lo anterior, el objetivo del presente estudio consistió en la evaluación del efecto del permanganato de potasio en la conservación de banano bocadillo con el fin de incrementar la vida poscosecha de este producto.

\section{MATERIALES Y MÉTODOS}

El estudio se llevó a cabo durante el primer semestre se 2012, para lo cual se colectaron frutos de bananito, clon Orito (Musa AA Simonds) en madurez fisiológica provenientes del departamento del Quindío, municipio de Calarcá. Los análisis de laboratorio se realizaron en el laboratorio de Poscosecha de la Facultad de Agronomía de la Universidad Nacional de Colombia, Bogotá. 
Para evaluar el efecto del tipo de arcilla, dosis de arcilla y dosis de $\mathrm{KMnO}_{4}$ en la conservación de banano bocadillo, se utilizó un diseño en bloques completos al azar con arreglo factorial de tratamientos $(3 \times 3+1)$ donde los bloques correspondieron a los tipos de arcillas (montmorillonita, caolinita, vermiculita y zeolita; cuyas características se presentan en la tabla 1). El primer factor correspondió a las dosis de arcillas (0,5\%; $1,0 \%$ ó $1,5 \%$ de arcilla), el segundo factor consistió en las dosis de $\mathrm{KMnO}_{4}(0,5 \% ; 1,0 \%$ y $1,5 \%$ de $\mathrm{KMnO}_{4}$ ), además se incluyó un testigo absoluto, para un total de 10 tratamientos, cada uno con 4 repeticiones. $\mathrm{La}$ arcilla y el $\mathrm{KMnO}_{4}$ se calcularon teniendo en cuenta el peso fresco de los frutos, se mezclaron uniformemente y se empacaron en sobres de celulosa (capacidad $5 \mathrm{~g}$ ) y luego se ubicaron en las unidades experimentales (UE).

Cada una de las 40 UE estuvo compuesta por cinco frutos (300 g de fruta fresca aproximadamente), que fueron empacados en bolsas de polietileno de baja densidad. Los frutos se almacenaron en condiciones ambientales a $18^{\circ} \mathrm{C}$ y humedad relativa entre $70 \%$ y $80 \%$. Las características fisicoquímicas de los frutos al inicio del experimento se presentan en la tabla 2 . Se realizaron mediciones de variables poscosecha a los 8 y 16 días después del inicio de los tratamientos (ddt).

Las variables evaluadas fueron: pérdida de peso (\%) a través de la medición de peso fresco con balanza (Ohaus, Ohio, $\mathrm{OH}$ ) de precisión 0,0001 g; firmeza del fruto $(\mathrm{N})$ mediante el penetrómetro digital PCE-PTR200 (PCE Ibérica S.L., Albacete, España); el color se determinó con el uso del colorímetro Minolta en el espacio CIELab (Konica Minolta, Tokio), se realizaron lecturas en la sección ecuatorial de los frutos y se calculó el índice de color (IC) con base en lo propuesto por Hunter Lab $\left(\mathrm{IC}=\left(1.000^{*} \mathrm{a}\right) /\left(\mathrm{L}^{*} \mathrm{~b}\right)\right)$. La acidez total titulable (ATT; \% de acido málico) se determinó según lo indicado por Dadzie y Orchard (1997); los sólidos solubles totales (SST; ${ }^{\circ}$ Brix) mediante el uso del refractómetro digital Hanna (Hanna Instruments, Woonsocket, RI) de rango 0 a 85\% a $20^{\circ} \mathrm{C}$, de acuerdo a Dadzie y Orchard (1997); la relación de madurez se calculó como la relación entre SST/ATT.

Los datos obtenidos cumplieron con los supuestos de normalidad (Shapiro-Wilk) y homogeneidad de varianzas, por tanto, se realizó un análisis de varianza (Anava) y prueba de comparación múltiple de Tukey a un nivel de confianza del $95 \%(P \leq 0,05)$. Todo mediante la utilización del software SAS v. 9.2.

\section{RESULTADOS Y DISCUSIÓN}

El índice de color (IC) en bananito tiende a incrementar con el almacenamiento desde valores negativos (color verde) a valores positivos (color amarillo). Se presentaron diferencias significativas $(P \leq 0,001)$ a los $16 \mathrm{ddt}$ entre las diferentes arcillas y dosis de arcilla y $\mathrm{KMnO}_{4}(P \leq 0,05)$. La arcilla que contribuyó a un menor IC a los 16 ddt

Tabla 1. Características de diferentes arcillas.

\begin{tabular}{|c|c|c|c|c|c|c|}
\hline Arcilla & Fórmula & Tipo & $\begin{array}{c}\text { CIC } \\
\left(\text { meq } 100 g^{-1}\right)\end{array}$ & $\begin{array}{l}\text { Densidad } \\
\left(\mathrm{g} \mathrm{cm}^{3}\right)\end{array}$ & $\begin{array}{c}\text { SE } \\
\left(\mathrm{m}^{2} \mathrm{~g}^{-1}\right)\end{array}$ & Fuente \\
\hline Zeolita & $\left(\mathrm{AlO}_{4}^{-}\right)_{5}\left(\mathrm{SiO}_{4}^{-}\right)_{4}$ & & $2-2,7$ & 1,20 & $12,60-500,12$ & 1 \\
\hline Montmorillonita & $(\mathrm{NaCa})_{0,3}(\mathrm{AlMg})_{2} \mathrm{Si}_{4} \mathrm{O}_{10}(\mathrm{OH})_{2}$ & $2: 1$ & 125 & 1,24 & 61 a 138 a 760 & 1 \\
\hline Caolinita & $\mathrm{Al}_{2} \mathrm{Si}_{2} \mathrm{O}_{5}(\mathrm{OH})_{4}$ & $1: 1$ & 3 a 15 & 1,04 & 5 a 40 & 2 \\
\hline Vermiculita & $(\mathrm{MgFe})_{3}\left[(\mathrm{SiAl})_{4} \mathrm{O}_{10}\right][\mathrm{OH}]_{2} 4 \mathrm{H}_{2} \mathrm{O}$ & $2: 1$ & 100 a 150 & 0,29 & 760 & 2 \\
\hline
\end{tabular}

Fuente: 1) Hang y Brindley (1970); 2) Miranda-Treviño y Coles (2003).

CIC, capacidad de intercambio catiónico; SE, superficie especifica. 
Tabla 2. Variables físico-químicas de frutos de bananito al inicio del experimento, los valores a continuación del promedio corresponden a la desviación estándar.

\begin{tabular}{|c|c|c|c|c|}
\hline Índice de color (IC) & $\begin{array}{c}\text { Sólidos solubles } \\
\text { totales }\left({ }^{\circ} \text { Brix }\right)\end{array}$ & Firmeza (N) & $\begin{array}{c}\text { Acidez total titulable } \\
(\%)\end{array}$ & RM (SST/ATT) \\
\hline$-13,28 \pm 1,18$ & $6,53 \pm 1,38$ & $74,86 \pm 19,55$ & $0,31 \pm 0,02$ & $20,89 \pm 1,25$ \\
\hline
\end{tabular}

fue vermiculita $(-0,01)$, mientras que este parámetro fue mayor en caolinita $(4,72)$ (figura $1 \mathrm{~A}$ ). La dosis de $0,5 \%$ de arcilla y $0,5 \%$ de $\mathrm{KMnO}_{4}$ presentó el mayor IC a los 16 ddt (7,72), mientras que $1 \%$ arcilla $+1,5 \% \mathrm{KMnO}_{4}$ mostró el menor IC $(-0,64)$ (tabla 3), es decir que hay mayor eficiencia en la conservación de bananito cuando el $\mathrm{KMnO}_{4}$ se encuentra en mayor proporción con respecto al carrier, siempre que el carrier presente menor densidad aparente favoreciendo el incremento del área de contacto etileno-absorbente (Wills y Warton, 2004).

El cambio de color que ocurre en la corteza durante la maduración de banano y bananito, es debido a la degradación de clorofilas y desenmascaramiento de carotenoides en los plastidos (Clendennen, 1997; Yang et al., 2011). En bananito existe una mayor concentración de clorofila a y b en los primeros días de almacenamiento, posteriormente éstas se degradan a clorofila a y feoforbida, carotenoides y xantofilas, debido a la acción de enzimas clorofilasas que conllevan a desarrollo de coloración amarilla en la corteza, debido al desenmascaramiento y síntesis de novo de carotenoides (Castro et al., 2007).

Con los resultados anteriores se observa que existe una respuesta diferencial de las arcillas usadas como carriers de $\mathrm{KMnO}_{4}$ en la velocidad de desarrollo de color en los frutos (Santosa et al., 2010; Pereira et al., 2009), en donde la vermiculita presenta un mejor comportamiento en la conservación de bananito. Wills y Warton (2004) proponen que los carriers más eficientes en reducir los niveles de etileno y retrasar la madurez de frutas y hortalizas, son aquellos con características de baja densidad aparente (DA) y alta capacidad de absorber $\mathrm{KMnO}_{4}$, lo cual depende de la capacidad de intercambio catiónico (CIC) y de la superficie específica (SE) de la arcilla. De esta forma, la vermiculita presenta la menor DA $\left(0,29 \mathrm{~g} \mathrm{~cm}^{-3}\right)$, mayor CIC (760 meq $\left.100 \mathrm{~g}^{-1}\right)$ y SE (tabla 1), con lo cual se aumenta el área de contacto entre el $\mathrm{KMnO}_{4}$ y el etileno, disminuyendo la acumulación de esta hormona, y por tanto, las reacciones metabólicas que conllevan a la maduración en poscosecha (Buthia et al., 2011).

Durante el almacenamiento todas las arcillas generaron un comportamiento lineal en la pérdida de peso (PP), sin presentar diferencias significativas $(P>0,05)$ en las evaluaciones realizadas $(0,8$ y 16 ddt). No obstante, a los $16 \mathrm{ddt}$ la PP fue menor en zeolita $(14,28 \%)$ y mayor en caolinita (15,91\%) (figura 1B). Las dosis de arcilla y $\mathrm{KMnO}_{4}$ presentaron diferencias significativas $(P \leq 0,0001)$ (tabla 3$)$. La PP fue mayor en el testigo absoluto, y menor en $1 \%$ arcilla $+1 \% \mathrm{KMnO}_{4}(12,62 \%)$.

En frutos de banano, la PP es ocasionada por la respiración, transpiración (Santosa et al., 2010) y lixiviación de solutos debido al incremento de la permeabilidad de membranas (Wade, 1995). Los resultados muestran que existe un efecto positivo del $\mathrm{KMnO}_{4}$ indiferentemente de la dosis de este o de su carrier, en la reducción de la PP. Lo anterior puede ser debido a la oxidación del etileno por parte del $\mathrm{KMnO}_{4}$, lo cual a su vez puede disminuir la acción de enzimas relacionadas con la respiración y otros procesos durante la maduración (Buthia et al., 2011). Por otro lado, Sammi y Masud (2007) proponen que el $\mathrm{KMnO}_{4}$ al oxidar el etileno produce $\mathrm{CO}_{2}$ y $\mathrm{H}_{2} \mathrm{O}$, lo cual genera al interior de los empaques una atmósfera modificada con altas concentraciones de $\mathrm{CO}_{2}$ y de humedad que reduce la transpiración. 
Los sólidos solubles totales (SST) incrementaron durante el almacenamiento (figura $1 \mathrm{C}$ ). Solo se presentaron diferencias significativas $(P \leq 0,001)$ a los $16 \mathrm{ddt}$ entre las diferentes arcillas, donde la caolinita alcanzó los mayores SST (19,2 ${ }^{\circ}$ Brix), y vermiculita mostró los menores valores $(15,79$ ${ }^{\circ}$ Brix). Las dosis de arcilla y permanganato mos- traron diferencias significativas $(P \leq 0,0001)$, siendo mayor en el testigo absoluto $\left(20,63^{\circ} \mathrm{Brix}\right)$ y menor en $1 \%$ de arcilla $+1,5 \%$ de $\mathrm{KMnO}_{4}(12,08$ ${ }^{\circ}$ Brix) (tabla 3). Los resultados obtenidos en este estudio se encuentran dentro del rango reportado por Chang-Yuen y Sáenz (2005), los cuales aseguran que en banano los SST incrementan

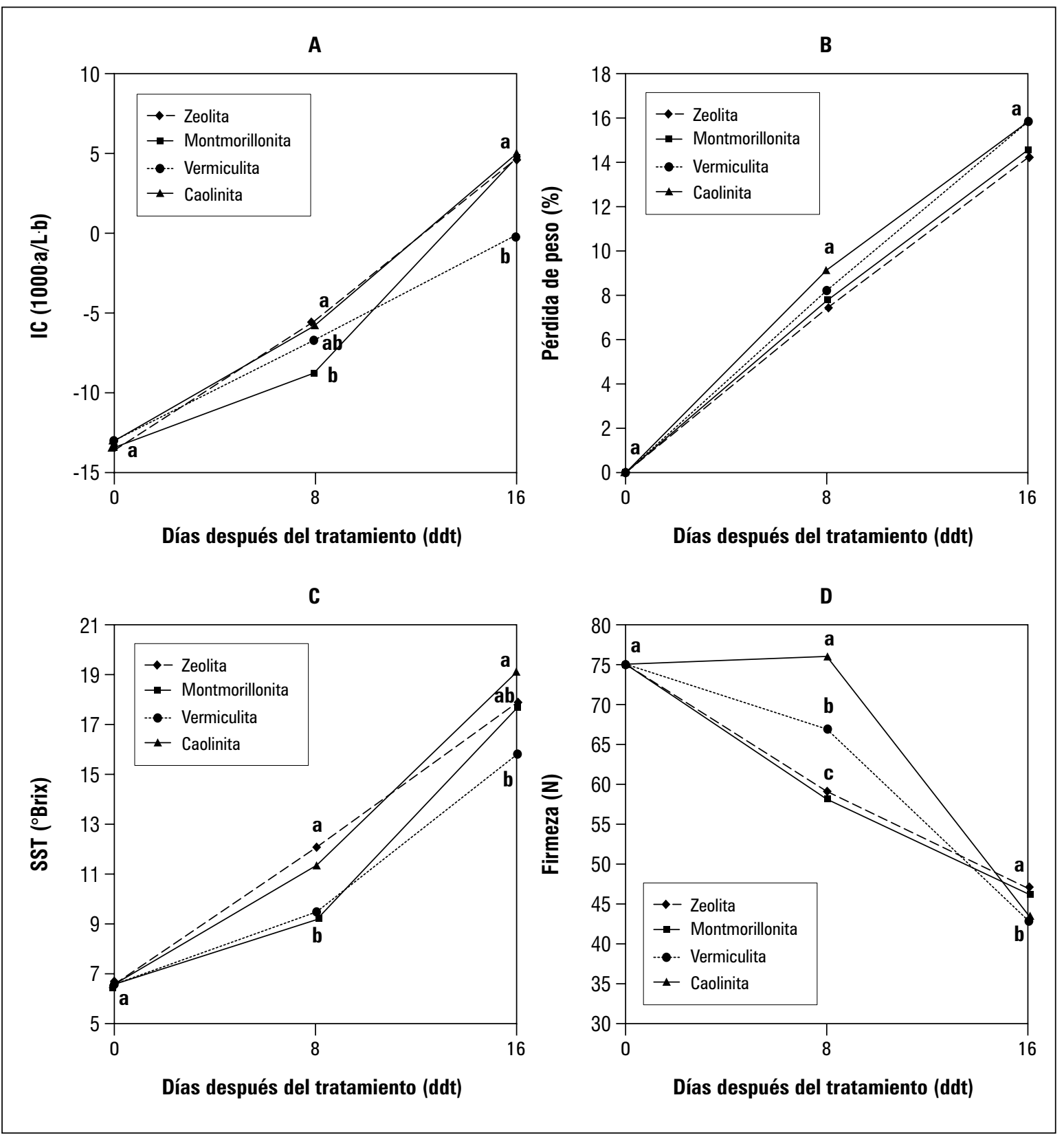

Figura 1. Efecto de las arcillas zeolita, montmorillonita, caolinita y vermiculita usadas como carrier de $\mathrm{KMnO}_{4}$ sobre: A. Índice de color (IC), B. Pérdida de peso (PP), C. Sólidos solubles totales (SST), D. Firmeza (F), E. Acidez total titulable (ATT) y F. Relación de madurez (RM) de frutos de bananito. Promedios con letras distintas indican diferencia significativa en cada punto de muestreo según la prueba de Tukey $(P \leq 0,05)$. (Continúa en la siguiente página) 


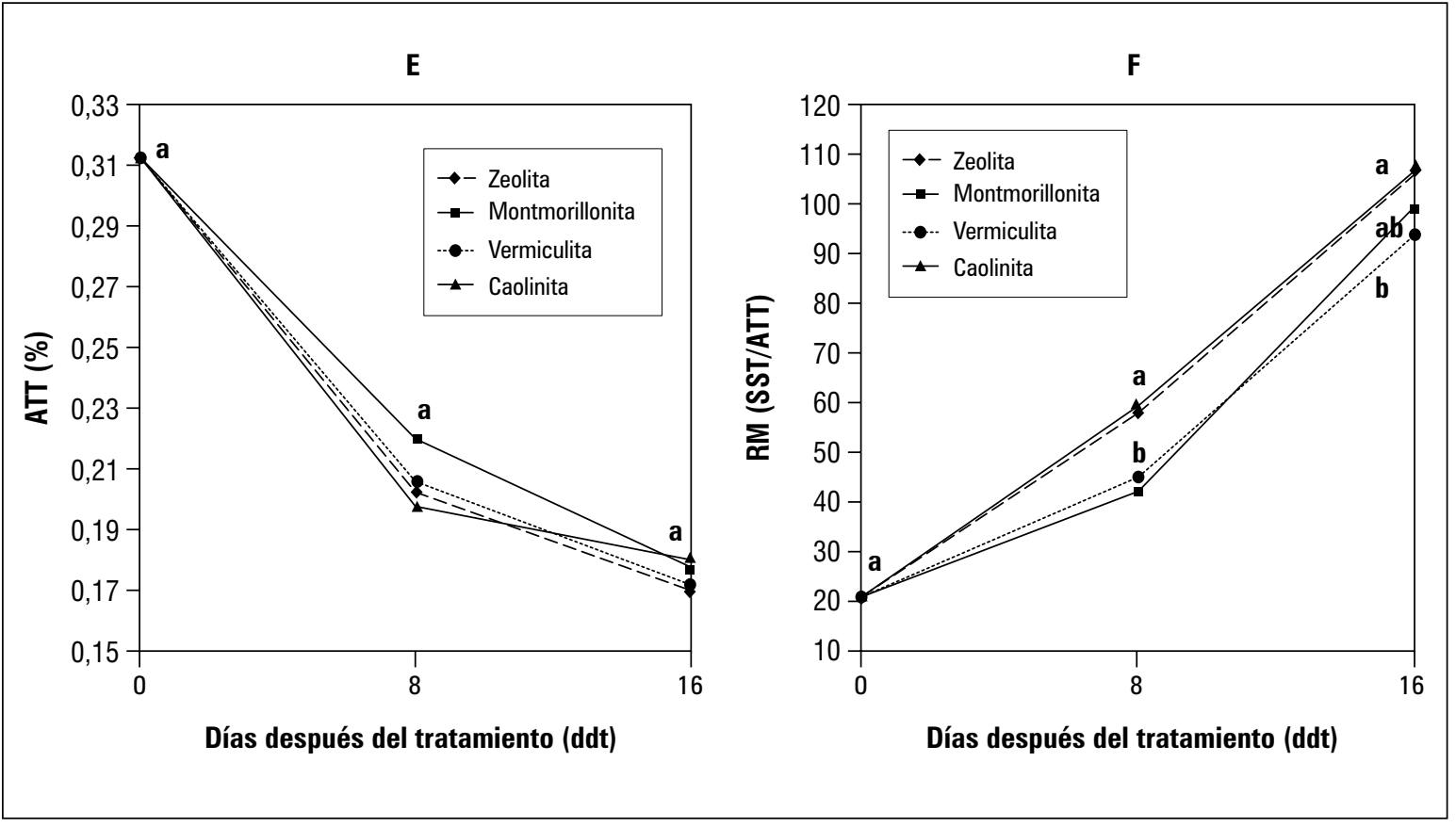

Figura 1. Efecto de las arcillas zeolita, montmorillonita, caolinita y vermiculita usadas como carrier de $\mathrm{KMnO}_{4}$ sobre: A. Índice de color (IC), B. Pérdida de peso (PP), C. Sólidos solubles totales (SST), D. Firmeza (F), E. Acidez total titulable (ATT) y F. Relación de madurez (RM) de frutos de bananito. Promedios con letras distintas indican diferencia significativa en cada punto de muestreo según la prueba de Tukey $(P \leq 0,05)$.

desde $4^{\circ}$ Brix en madurez fisiológica hasta $20^{\circ}$ Brix a los $8 \mathrm{~d}$ de almacenamiento a $20^{\circ} \mathrm{C}$.

El incremento de los SST en banano y bananito durante el almacenamiento es debido a la degradación del almidón y acumulación de sacarosa en la pulpa de los frutos, los cuales son reportados como los principales procesos que ocurren durante la maduración del fruto de banano (Wen et al., 2011). Choudhury et al. (2009) reportan una degradación de almidón en frutos de banano y plátano entre el 58 y $70 \%$ en los primeros días de almacenamiento en condiciones ambientales $\left(18-20^{\circ} \mathrm{C}\right)$. Por su parte, Palomer et al. (2005), proponen que el incremento de los SST en bananito es debido a una excesiva transformación de almidón en azúcares, lo cual es mediado por varias enzimas como sacarosa fosfato sintasa (SPS), sacarosa sintasa (SS), invertasa ácida (AI) e invertasa neutra (NI), de las cuales la SPS ha sido considerada la enzima clave en la acumulación de sacarosa en frutos de banano, alcan- zando su mayor actividad (200-300 $\mathrm{mmol} \mathrm{h}^{-1} \mathrm{~g}^{-1}$ FW) entre los 10 y 20 d después de la madurez fisiológica del banano, lo cual corresponde a lo encontrado en el presente experimento teniendo en cuenta el contenido de SST (Wen et al., 2011). Otro factor que puede influir en el incremento de los SST es la producción de polisacáridos como, rhamnosa, arabinosa, xilosa y glucosa después de la hidrolisis de la fracción péctica (Payasi y Sanwal, 2003).

La arcilla que se comportó como mejor carrier del $\mathrm{KMnO}_{4}$ fue vermiculita, mientras que caolinita mostró la menor eficiencia en reducir el incremento de los SST, lo cual puede estar correlacionado con sus características fisicoquímicas, como, menor CIC (3-15 meq $\left.100 \mathrm{~g}^{-1}\right)$ y SE (5-40 $\mathrm{m}^{2} \mathrm{~g}^{-1}$; tabla 1). Los SST presentaron tendencia a incrementarse durante el almacenamiento, no obstante, la tasa de incremento de SST fue afectada por la dosis de $\mathrm{KMnO}_{4}$ y arcilla, lo cual corresponde a lo reportado por Buthia et al. (2011) 


\begin{tabular}{|c|c|c|c|c|c|c|c|c|c|c|c|c|c|c|}
\hline \multirow[b]{2}{*}{$\mathrm{ddt}$} & \multicolumn{2}{|c|}{ Dosis (\%) } & \multirow{2}{*}{\multicolumn{2}{|c|}{$\begin{array}{l}\text { Índice de } \\
\text { color } \\
\text { (IC) }\end{array}$}} & \multirow{2}{*}{\multicolumn{2}{|c|}{$\begin{array}{c}\text { Pérdida de } \\
\text { peso } \\
(\%)\end{array}$}} & \multirow{2}{*}{\multicolumn{2}{|c|}{$\begin{array}{l}\text { Sólidos } \\
\text { solubles } \\
\text { totales } \\
\left.{ }^{\circ} \text { Brix }\right)\end{array}$}} & \multirow{2}{*}{\multicolumn{2}{|c|}{$\begin{array}{c}\text { Firmeza } \\
\text { (N) }\end{array}$}} & \multirow{2}{*}{\multicolumn{2}{|c|}{$\begin{array}{c}\text { Acidez total } \\
\text { titulable } \\
(\%)\end{array}$}} & \multirow{2}{*}{\multicolumn{2}{|c|}{$\begin{array}{c}\text { RM } \\
\text { (SST/ATT) }\end{array}$}} \\
\hline & \multirow{2}{*}{$\begin{array}{c}\text { Arcilla } \\
\text { 0,5 }\end{array}$} & \multirow{2}{*}{$\begin{array}{c}\mathrm{KMnO}_{4} \\
0,5\end{array}$} & & & & & & & & & & & & \\
\hline & & & $-2,09$ & a & 7,73 & $\mathrm{~cd}$ & 16,87 & a & 34,62 & $\mathrm{~h}$ & 0,228 & $a b$ & 74,80 & a \\
\hline & 0,5 & 1,0 & $-5,22$ & b & 9,42 & $b$ & 11,32 & b & 62,66 & ef & 0,217 & $b c$ & 52,24 & de \\
\hline & 0,5 & 1,5 & $-9,82$ & $f$ & 7,04 & cde & 12,90 & $b$ & 55,55 & $\mathrm{fg}$ & 0,197 & $\mathrm{~cd}$ & 65,61 & $a b$ \\
\hline & 1,0 & 0,5 & $-5,12$ & $b$ & 7,05 & cde & 8,40 & c & 52,85 & g & 0,215 & $b c$ & 39,53 & $\mathrm{fg}$ \\
\hline 0 & 1,0 & 1,0 & $-6,64$ & $\mathrm{~cd}$ & 7,19 & cde & 12,90 & $b$ & 74,08 & bc & 0,247 & a & 52,29 & de \\
\hline 0 & 1,0 & 1,5 & $-8,01$ & e & 7,59 & $\mathrm{~cd}$ & 6,07 & $d$ & 79,92 & bc & 0,185 & d & 33,24 & g \\
\hline & 1,5 & 0,5 & $-7,37$ & de & 6,46 & e & 8,70 & c & 74,12 & $a b$ & 0,187 & $d$ & 46,68 & def \\
\hline & 1,5 & 1,0 & $-6,26$ & bcd & 6,78 & de & 11,77 & $b$ & 58,25 & efg & 0,187 & $d$ & 62,44 & bc \\
\hline & 1,5 & 1,5 & $-7,37$ & de & 7,90 & C & 8,92 & c & 84,90 & a & 0,200 & $c d$ & 44,59 & ef \\
\hline & 0,0 & 0,0 & $-5,79$ & bcd & 15,01 & a & 11,18 & $b$ & 65,41 & de & 0,202 & bcd & 57,19 & $\mathrm{~cd}$ \\
\hline & 0,5 & 0,5 & 7,72 & $\mathrm{a}$ & 13,82 & $\mathrm{~cd}$ & 19,8 & $a b$ & 24,46 & $f$ & 0,179 & $a b c$ & 110,38 & a \\
\hline & 0,5 & 1,0 & 5,63 & $a b$ & 16,06 & $b c$ & 18,83 & $a b c$ & 46,59 & C & 0,170 & bcd & 112,68 & a \\
\hline & 0,5 & 1,5 & 3,19 & $a b$ & 13,05 & $d$ & 18,9 & $a b c$ & 48,89 & $b c$ & 0,177 & $a b c$ & 110,04 & a \\
\hline & 1,0 & 0,5 & 3,52 & $a b$ & 17,07 & $b$ & 19,28 & $a b$ & 24,5 & $f$ & 0,170 & bcd & 115,14 & a \\
\hline 16 & 1,0 & 1,0 & 2,51 & $a b$ & 12,62 & $d$ & 15,75 & $d$ & 44,13 & $\mathrm{~cd}$ & 0,155 & $\mathrm{~cd}$ & 106,73 & $a b$ \\
\hline & 1,0 & 1,5 & $-0,64$ & $b$ & 15,35 & bcd & 12,08 & e & 70,65 & $\mathrm{a}$ & 0,149 & $d$ & 83,67 & c \\
\hline & 1,5 & 0,5 & 5,31 & $a b$ & 12,68 & $d$ & 15,6 & $d$ & 40,45 & $\mathrm{~cd}$ & 0,200 & a & 82,39 & $\mathrm{c}$ \\
\hline & 1,5 & 1,0 & 3,49 & a & 13,85 & $\mathrm{~cd}$ & 18,45 & $a b c$ & 29,6 & ef & 0,188 & $a b$ & 98,18 & $b$ \\
\hline & 1,5 & 1,5 & 2,51 & $a b$ & 14,2 & $\mathrm{~cd}$ & 17,78 & $\mathrm{bcd}$ & 36,23 & de & 0,170 & bcd & 108,27 & $\mathrm{a}$ \\
\hline & 0,0 & 0,0 & 2,09 & $a b$ & 25,16 & a & 20,63 & a & 69,38 & $\mathrm{a}$ & 0,2 & $\mathrm{a}$ & 106,55 & $a b$ \\
\hline
\end{tabular}

en zapote, Chaves et al. (2007) en guanábana y Choudhury et al. (2009) en banano, quienes encontraron una menor tasa de incremento a mayores dosis de $\mathrm{KMnO}_{4}$.

La firmeza de los frutos de bananito tiende a disminuir con el almacenamiento. Las arcillas presentaron diferencias significativas $(P \leq 0,05)$ en las evaluaciones realizadas, en donde la firmeza de los frutos almacenados en presencia de vermiculita y caolinita como carrier disminuye lentamente hasta los $8 \mathrm{ddt}$, a partir de lo cual ocurre una rápida disminución hasta los $16 \mathrm{ddt}$, tiempo en el cual fue mayor en zeolita $(46,86 \mathrm{~N})$ y menor en vermiculita $(42,68 \mathrm{~N})$ (figura 1D). Las do- sis de arcilla y $\mathrm{KMnO}_{4}$ presentaron diferencias significativas $(P \leq 0,0001)$, donde $1 \%$ de arcilla $+1,5 \% \mathrm{KMnO}_{4}$ mostró menor disminución en el periodo de almacenamiento $(5,62 \%$, de 74,86 $\mathrm{N}$ a $70,65 \mathrm{~N}$ ). Los tratamientos con menor proporción de $\mathrm{KMnO}_{4}(0,5 \%)$ presentaron la menor firmeza a los 16 ddt (tabla 3).

La pérdida de firmeza y ablandamiento de frutos en bananito puede ser debida a tres procesos, los cuales comprenden la degradación de almidón e incremento en los SST en la pulpa (correlación firmeza y SST $r=-0,72, P \leq 0,005$ ), solubilización de sustancias pécticas y pérdida de agua del fruto, tal como se reporta para 
frutos de banano (Chang-Yuen y Sáenz, 2005; Torres et al., 2012). Lohani et al. (2004) aseguran que la principal causa del ablandamiento en banano son los cambios en el componente de pectinas en la lamela media de la pared celular, los cuales son atribuidos a síntesis de hidrolasas como poligalacturonasa (PG), pectinmetilesterasa (PME), pectato liasa (PL) y celulasas en la pulpa, sin embargo, no se sabe si éstas mismas enzimas actúan en la misma proporción en la corteza de los frutos de banano (Imsabai et al., 2006). La actividad de estas enzimas depende del estado de maduración de los frutos y es estimulada por el etileno, por lo tanto, el uso de $\mathrm{KMnO}_{4}$ afecta de manera indirecta la expresión de dichas enzimas al reducir los niveles de etileno en la atmósfera, favoreciendo la conservación de los frutos de bananito.

Las diferentes arcillas no mostraron diferencias significativas $(P>0,05)$ en la acidez total titulable (ATT) durante las evaluaciones realizadas. Éste parámetro poscosecha disminuye con la maduración del fruto de bananito de tal forma que en el inicio del experimento la ATT fue de $0,31 \%$ de ácido málico, mientras que a los 16 ddt la ATT se redujo hasta $0,17 \%$ (figura $1 \mathrm{E}$ ). Las dosis de arcillas y $\mathrm{KMnO}_{4}$ presentaron diferencias significativas $(P \leq 0,0001)$. En este estudio se encontró que la ATT en el testigo absoluto presentó la mayor ATT a los 16 ddt (0,20\% ácido málico), mientras que el tratamiento $1 \%$ arcilla $+1,5 \% \mathrm{KMnO}_{4}$ mostró el menor valor $(0,15 \%$; tabla 3$)$.

El comportamiento de la ATT en bananito encontrado en este estudio no corresponde a lo reportado por Chang-Yuen y Sáenz (2005), quienes aseguran que la ATT de los tejidos de la pulpa de banano de la mayoría de cultivares e híbridos de banano muestra grandes aumentos durante la maduración, alcanzando valores de 0,36\%. Por otro lado, Buthia et al. (2011) reportan disminución de la ATT en zapote, en donde frutos tratados con $\mathrm{KMnO}_{4}$ muestran significativamente mayor ATT indiferentemente del estado de madurez. A partir de lo anterior, Torres et al. (2012) reportan que el ácido málico puede ser sustrato respiratorio, no obstante, frutos climatéricos como el banano y mango parecen no usarlo durante el pico respiratorio. Sin embargo, existe la posibilidad que en bananito, dicho ácido participe en la respiración, teniendo en cuenta la alta perecibilidad de este fruto, por tanto se deben realizar más estudios para evaluar el comportamiento de los ácidos presentes en bananito. También, es posible que los ácidos orgánicos sean transformados a azúcares nuevamente en el proceso de gluconeogénesis, disminuyendo la ATT e incrementando los SST (Sinha et al., 2012).

La relación de madurez incrementa con el almacenamiento, siendo menor en vermiculita $(93,58)$ a los 16 ddt y mayor en caolinita $(106,5)$ (figura $1 \mathrm{~F}$ ), presentándose diferencias significativas $(P \leq 0,05)$ entre arcillas. En las dosis de arcillas y permanganato hubo diferencias significativas $(P<0,0001)$, siendo mayor en el tratamiento $0,5 \%$ arcilla $+0,5 \% \mathrm{KMnO}_{4}$ y menor $1,5 \%$ arcilla $+0,5 \% \mathrm{~K} \mathrm{MnO}_{4}$ seguido por el $1 \%$ arcilla $+1,5 \% \mathrm{KMnO}_{4}$ sin presentar diferencias significativas (tabla 3 ).

Los resultados permiten afirmar que los frutos tratados con $\mathrm{KMnO}_{4}$ en mezcla con vermiculita presentaron menor SST y mayor ATT, es decir que dicha arcilla contribuyó a la disminución de la velocidad de maduración de frutos de bananito, presentando mejores características organolépticas para el consumo. Chaves et al. (2007) reportaron disminución de la relación de madurez en guanábana con el uso de $\mathrm{KMnO}_{4}$, de tal forma que con dosis entre $9 \%$ y $12 \%$ de $\mathrm{KMnO}_{4}$ la madurez de consumo se alcanzó a los $12 \mathrm{~d}$.

\section{CONCLUSIONES}

La arcilla que presentó un mejor comportamiento como carrier de $\mathrm{KMnO}_{4}$ en la conservación de banano bocadillo fue vermiculita, debido a que mantuvo por más tiempo el color verde, contribuyó a disminuir la conversión de almidón en 
azúcares, y tuvo una menor relación de madurez, esto puede ser debido a sus características físico-químicas como mayor CIC, SE y densidad. Por otro lado, mayores concentraciones de $\mathrm{KMnO}_{4}(1,5 \%$ con base en peso fresco de los frutos) con respecto al carrier (1\%) retardan la ma- durez de bananito al disminuir el IC, pérdida de peso, SST, relación de madurez e incrementar la firmeza. Lo anterior puede ser una herramienta útil durante el almacenamiento y transporte de banano bocadillo en condiciones ambientales.

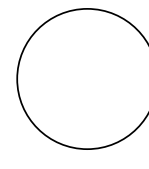

Agronet. 2012. Análisis estadísticas del bananito. En: http://www.agronet.gov.co; consulta: septiembre de 2012.

Ayoub, J., M. Driver, H. Kim, A. Barret e I. Taub, 1976. Use of ethylene absorbers in extending produce shelf life. En: http://agraconew.com. 8 p.; consulta: septiembre de 2012.

Brackmann, A., R. Giehl, S. Freitas, A. Eisermann y A. Mello. 2006. Uso de filmes de polietileno e absorção de etileno para o transporte refrigerado de maçã 'Gala'. Ciências Agrárias 27 (3), 423-428.

Bhutia, W., R. Pal, S. Sen y S. Jha. 2011. Response of different maturity stages of sapota (Manilkara achras Mill.) cv. Kallipati to in-package ethylene absorbent. J. Food Sci. Technol. 48(6), 763-768.

Bonilla, M., P. Arias, L. Landínez, J. Moreno, F. Carozo y M. Suárez. 2009. Agenda prospectiva de investigación y desarrollo tecnológico para la cadena productiva de la uchuva en fresco para exportación en Colombia. Ministerio de Agricultura y Desarrollo Rural, Bogotá.

Brody, A., E. Strupinsky y L. Kline. 2002. Active packaging for food applications. CRC Press, London.

Campos, J., P. Hasegawa, E. Purgatto, F. Lajolo y B. Cordenunsi. 2007. Qualidade póscolheita de nêsperas submetidas ao armazenamento sob baixa temperatura e atmosfera modificada. Ciência e Tecnologia de Alimentos 27(2), 401-407.

Castro, M. 2001. Efectos de tratamientos postcosecha en la actividad enzimática de la clorofilasa del banano bocadillo (Musa Accuminata). Tesis de maestría. Facultad de Agronomía, Universidad Nacional de Colombia, Bogotá.

Castro, B. y L. Restrepo. 2004. Efectos de tratamientos postcosecha sobre la prolongación de la maduración del baby banano (Musa accuminata). pp. 273-

\section{REFERENCIAS BIBLIOGRÁFICAS}

274. Memorias XVI Reunión Internacional Acorbat. Oxaca, México.

Castro, B., G. Jerz, P. Winterhalter y R. Restrepo. 2007. Degradación de la clorofila en la corteza del baby banano (Musa acuminata) durante diferentes estados de maduración. Memorias VIII Congreso Nacional del Color. 19-21 Septiembre, 2007. Madrid.

Chang-Yuen, K. y V. Sáenz. 2005. Efecto del 1-Metil-ciclopropeno (1-MCP) en la maduración de banano. Agron. Costarr. 29(3), 211-220.

Chauhan, O., P. Raju, D. Dasgupta y A. Bawa. 2006. Modified atmosphere packaging of banana (cv. Pachbale) with ethylene, carbon dioxide and moisture scrubbers and effect on its ripening behaviour. Amer. J. Food Technol. 1(2), 179-189.

Chaves, M., R. Bonomo, A. Silva, L. Santos, B. Carvalho, T. Souza, G. Gomes y R. Soares. 2007. Use of potassium permanganate in the sugar apple post-harvest preservation. Cienc. Tecnol. Aliment. 5(5), 346-351.

Choudhury, S., S. Roy y D. Sengupta. 2009. A comparative study of cultivar differences in sucrose phosphate synthase gene expression and sucrose formation during banana ripening. Postharvest Biol. Technol. 54, 15-24.

Clendennen, S. y D. May. 1997. Differential gene expression in ripening banana fruit. Plant Physiol. $115,463-469$.

Dadzie, B. y J. Orchard. 1997. Evaluación rutinaria postcosecha de híbridos de bananos y plátanos: criterios y métodos. Guías técnicas Inibap 2. Instituto Internacional de Recursos Fitogenéticos, Roma, Italia; Red Internacional para el Mejoramiento del Banano y el Plátano, Montpellier, Francia.

Dash, S., S. Patel y B. Mishra. 2009. Oxidation by permanganate: synthetic and mechanistic aspects. Tetrahedron. 65, 709-739. 
Espinal, C., H. Martínez y Y. Peña. 2005. La cadena de los frutales de exportación en Colombia. Ministerio de Agricultura y Desarrollo Rural. Observatorio Agrocadenas Colombia. En: http://www.fondohortifruticola.com.co. 67 p.; consulta: noviembre de 2011.

Gutiérrez, J. 1997. Efecto del uso del absorbente de etileno en el almacenamiento de banano bocadillo (Musa acuminata). Trabajo de grado. Departamento de Ingeniería Agrícola, Facultad de Ingeniería, Universidad Nacional de Colombia, Bogotá.

Hang, P. y G. Brindley. 1970. Methylene blue absorption by clay minerals. Determination of surface areas and cation exchange capacities (clayorganic studies XVIII). Clays Clay Minerals 18, 203-212.

Illeperuma, C. y P. Jayasuriya. 2002. Prolonged storage of 'Karuthacolomban' mango by modified atmosphere packaging at low temperature. J. Hort. Sci. Biotechnol. 77(2), 153-157.

Illeperuma, C. y C. Nikapitiya. 2002. Extension of the postharvest life of 'Pollock' avocado using modified atmosphere packaging. Fruits 57 (5/6), 287-295.

Imsabai, W., S. Ketsa y W. Van Doorn. 2006. Physiological and biochemical changes during banana ripening and finger drop. Postharvest Biol. Technol. 39, 211-216.

Jiang. Y., D. Joyce y A. Macnish. 1999. Extension of the shelf life of banana fruit by 1-methylcyclopropene in combination with polyethylene bags. Postharvest Biol. Technol. 16, 187-193.

Kesari, R., P. Trivedi y P. Nath. 2007. Ethylene-induced ripening in banana evokes expression of defense and stress related genes in fruit tissue. Postharvest Biol. Technol. 46, 136-143.

Legiscomex. 2008. Exportaciones de bananito. En: www. legiscomex.com. 16 p.; consulta: marzo de 2012.

Lohani, S., P. Trivedi y P. Nath. 2004. Changes in activities of cell wall hydrolases during ethylene-induced ripening in banana: effect of $1-\mathrm{MCP}, \mathrm{ABA}$ and IAA. Postharvest Biol. Technol. 31, 119-126.

Morales, N. 2002. Comportamiento fisiológico, químico y sensorial de banana bocadillo (Musa acuminata L.) producido en algunas zonas del departamento del Tolima. Trabajo de grado. Departamento de Química, Facultad de Ciencias, Universidad Nacional de Colombia, Bogotá.

Miranda-Treviño, J. y C. Coles. 2003. Kaolinite properties, structure and influence of metal retention on pH. Appl. Clay Sci. 23, 133-139.
Palomer, X., I. Roing-Villanova, D. Grima-Calvo y M. Venderll. 2005. Effects of nitrous oxide $\left(\mathrm{N}_{2} \mathrm{O}\right)$ treatment on the postharvest ripening of banana fruit. Postharvest Biol. Technol. 36, 167-175.

Payasi, A. y G. Sanwal. 2003. Pectate lyase activity during ripening of banana fruit. Phytochem. 63, 243-248.

Pereira, D., L. Chamhum, D. Lopes, P. Cecon y A. Rocha. 2009. Potassium permanganate effects in postharvest conservation of the papaya cultivar Sunrise Golden. Pesq. Agropec. Bras. 44 (7), 669-675.

Saltveit, M. 1999. Effect of ethylene on quality of fresh fruits and vegetables. Postharvest Biol. Technol. 15, 279-292

Sammi, S. y T. Masud. 2007. Effect of different packaging systems on storage life and quality of tomato (Lycopersicon esculentum var. Rio Grande) during different ripening stages. Internet J. Food Safety 9, 37-44.

Santosa, E., W. Widodo y C. Kholidi. 2010. The use of clay as potassium permanganate carrier to delay the ripening of Raja Bulu Banana. J. Hort. Indonesia 1(2), 89-96.

Sinha, N., J. Sidhu, J. Barta., J. Wu y M. Cano. 2012. Handbook of fruits and fruit processing. $2^{\text {nd }} \mathrm{ed}$ Wiley-Blackwell, New York, NY.

Torres, T., S. Beserra, B. Cordenunsi, F. Gozzo, E. Pilau, F. Lajolo y J. Oliveira. 2012. Proteomic analysis of banana fruit reveals proteins that are differentially accumulated during ripening. Postharvest Biol. Technol. 70, 51-58.

Wade, N. 1995. Membrane lipid composition and tissue leakage of pre- and early-climacteric banana fruit. Postharvest Biol. Technol. 5, 139-147.

Wen, L., S. Yuanzhi., C. Weixin y J. Wenjun. 2011. The effects of harvest maturity on storage quality and sucrose-metabolizing enzymes during Banana ripening. Food Bioprocess Technol. 4, 1273-1280.

Wills, R. y M. Warton. 2004. Efficacy of potassium permanganate impregnated into alumina beads to reduce atmospheric ethylene. J. Amer. Soc. Hort. Sci. 129(3), 433-438.

Yang, X., J. Song, S. Fillmore, X. Pang y Z. Zhang. 2011. Effect of high temperature on color, chlorophyll fluorescence and volatile biosynthesis in green-ripe banana fruit. Postharvest Biol. Technol. 62, 246-257.

Zhang, M., S. Tian, Z. Zhu, Y. Xu y G. Oin. 2002. Effects of 1-methylcyclopropene (1-MCP) on ripening and resistance of jujube (Zizyphus jujuba cv. Huping) fruit against postharvest disease. LWT - Food Sci. Technol. 45, 13-19. 